TITLE:

\title{
Observing the rotational diffusion of nanodiamonds with arbitrary nitrogen vacancy center configurations
}

AUTHOR(S):

Yoshinari, Yohsuke; Kalay, Ziya; Harada, Yoshie

CITATION:

Yoshinari, Yohsuke ...[et al]. Observing the rotational diffusion of nanodiamonds with arbitrary nitrogen vacancy center configurations. Physical Review B 2013, 88(23): 235206.

ISSUE DATE:

2013-12-16

URL:

http://hdl.handle.net/2433/182056

RIGHT:

(c) 2013 American Physical Society. 
PHYSICAL REVIEW B 88, 235206 (2013)

\title{
Observing the rotational diffusion of nanodiamonds with arbitrary nitrogen vacancy center configurations
}

\author{
Yohsuke Yoshinari, ${ }^{*}$ Ziya Kalay, ${ }^{\dagger}$ and Yoshie Harada ${ }^{\ddagger}$ \\ Institute for Integrated Cell-Material Sciences WPI-iCeMS, Kyoto University, Yoshida-Honmachi, Sakyo-ku, Kyoto 606-8501, Japan
}

(Received 23 August 2013; revised manuscript received 10 November 2013; published 16 December 2013)

\begin{abstract}
We present theoretical results on the relationship between the rotational diffusion coefficient of a nanodiamond undergoing Brownian motion and the configuration of nitrogen vacancy centers (NVCs) contained in the particle. Through exact calculations and simulations, we obtain the fluorescence intensity autocorrelation function that is measured in optically detected magnetic resonance experiments conducted at single-particle level. We relate the autocorrelation function to the rotational diffusion coefficient and discuss the influence of different NVC configurations on the outcome of measurements. We believe that our results can be useful in interpreting observations on nanodiamonds that contain multiple nitrogen vacancy centers.
\end{abstract}

DOI: 10.1103/PhysRevB.88.235206

\section{INTRODUCTION}

Random motion of microscopic particles in a fluid has been studied since the historic observations in the 18th and 19th centuries ${ }^{1}$ and the pioneering theoretical accounts given by Einstein $^{2}$ and Smoluchowski. ${ }^{3}$ In addition to its historic role as providing a key evidence for the discrete nature of molecules, quantitatively studying the apparently random motion of microscopic particles is an essential part of understanding many phenomena, and is an active research field. ${ }^{4-8}$ For instance, Edmond et al. ${ }^{9}$ demonstrated that simultaneous measurement of translational and rotational diffusion of optically anisotropic particle clusters in a supercooled colloidal fluid reveals important aspects of the dynamics near glass transition, such as the clear decoupling between translational and rotational degrees of freedom. It is usually less straightforward to observe the rotational motion of particles, as probed particles do not necessarily possess optical anisotropy. Techniques based on magnetic resonance, such as electron paramagnetic/spin resonance (EPR/ESR), ${ }^{10}$ have been successfully used in the pioneering observations of rotational diffusion of biological molecules, such as proteins in biological membranes. ${ }^{11}$

Recent advancements in the efficient production ${ }^{12}$ and observation of diamond nanocrystals with nitrogen vacancy centers $(\mathrm{NVCs})^{13}$ show that diamond nanocrystals can be promising probes in detecting rotational motion at the nanoscale. ${ }^{14-16} \mathrm{~A}$ negatively charged $\mathrm{NVC}$ in a diamond crystal is an atomic defect where the substitutional nitrogen and the adjacent vacancy are paired, creating an $S=1$ spin triplet quantum state. The spin state can be read out by optically detected magnetic resonance (ODMR) through the state dependent fluorescence intensity, even for a single NVC under ambient conditions. ${ }^{17}$ Such physical properties have been exploited to use diamond nanocrystals containing NVCs as sensors for not only magnetic field, ${ }^{18,19}$ but also for electric field ${ }^{20-22}$ and temperature, ${ }^{23-25}$ which are sensitive to spatial variations within a few tens of nanometers. The basic principle behind this is that the presence of a static magnetic field changes the ODMR spectrum drastically, enabling the precise determination of the ambient magnetic field in three dimensions. ${ }^{26-28}$ Conversely, this allows one to deduce the orientation of an NVC relative to the magnetic field, ${ }^{14,16}$ as the change in the spectrum depends on the angle
PACS number(s): 76.70.Hb, 45.20.dc, 05.40.Jc, 06.30.Gv

between the magnetic field and the NVC's principle axis. In an alternative approach, not based on continuous ODMR monitoring, Ledbetter et al..$^{29}$ have proposed NVC to be used as a gyroscope by measuring quantum Berry phase acquired by a mixing of spin states, and Maclaurin et al. ${ }^{30}$ applied quantum control principles to provide a way of using nanodiamonds for magnetometry and bioimaging.

In conventional microscopy, fluorescence measurements can be used in determining the orientation of a single molecule tagged with a fluorescent dye. This method is based on slightly defocusing the optics to reveal the polarization of the emitted light, altered by the electric dipole of the dye. ${ }^{31}$ However, the angular precision is often limited to a few tens of degrees, and the photobleaching of the dye prevents long-time measurements. On the other hand, an NVC is extremely photostable; it neither bleaches nor blinks, ${ }^{32}$ allowing one to trace a long term trajectory. Because the ODMR spectrum is sensitive to minute changes in the angle between the NVC and the external magnetic field, a diamond nanocrsytal containing $\mathrm{NVCs}$ is a promising probe to study rotation, making it possible to perform more quantitative measurements than the defocusing method.

In this paper, a theoretical analysis of the isotropic Brownian rotation of a nanodiamond containing single or multiple NVCs is presented, for the purpose of guiding the design of experiments to observe rotational diffusion. We first calculate the time trajectory of fluorescence intensity emitted from NVCs placed in a weak magnetic field, which can be directly measured in experiments. We then show that the autocorrelation function (ACF) of the fluorescence trajectory can be used to deduce the rotational diffusion constant. Our theoretical analysis indicates that the ACF can be expressed as a sum of decaying exponentials (which stems from the fact that the diffusion equation is solved in a finite space) with rate parameters given by $\ell(\ell+1)$ times the diffusion constant, where $\ell=2,4,6, \ldots$ We confirm this behavior by performing simulations, and clearly show that the decay of the ACF distinctly depends on the number and orientational configuration of NVCs in the crystal, implying that the observed signal is strongly correlated with the orientation configuration of NVCs. We also point out that the appropriate choice of the magnetic field amplitude is crucial for examining the dynamics without loss of signal coherency. 
The article is organized as follows. First, the theoretical formalism is described in Sec. II. In Sec. III, simulation results are presented along with comparisons between theoretical and computational results. Lastly, a discussion of our findings and our conclusions are given in Sec. IV. In order to avoid distraction from our main point, the details of several calculations are given in the appendices.

\section{THEORETICAL ANALYSIS}

\section{A. Energy eigenvalues and fluorescence intensity}

An NVC forms an $S=1$ spin triplet ground state. The axial crystal field and spin-spin interaction cause the three spin states of $S_{z}=-1,0,+1$ to split into $\left|S_{z}=0\right\rangle$ and $\left|S_{z}= \pm 1\right\rangle$ states separated by a zero field gap $D$, which is approximately equal to $2.87 \mathrm{GHz}$. The ground state spin Hamiltonian of a single $\mathrm{NVC}$ is expressed by

$$
\mathcal{H}=h\left[D\left(S_{z}^{2}-\frac{2}{3}\right)+\gamma \overrightarrow{\mathbf{S}} \cdot \vec{H}\right],
$$

where $h$ is the Planck constant, $\gamma=\gamma_{\mathrm{e}} / 2 \pi, \gamma_{\mathrm{e}}$ being the gyromagnetic ratio of the electron $(\gamma \approx 28.07 \mathrm{GHz} / \mathrm{T})$ and $\vec{H}$ is the applied magnetic field whose magnitude is $H_{0}$. In Eq. (1), the first term inside the brackets corresponds to the energy of the system in the absence of an external magnetic field, where $S_{z}$ stands for the spin operator, while the second term reflects the Zeeman interaction given by the inner product of the vector of spin operators $\overrightarrow{\mathbf{S}}=\left\{S_{x}, S_{y}, S_{z}\right\}$ and the vector $\vec{H}=\left\{H_{x}, H_{y}, H_{z}\right\}$. The Zeeman effect depends on the angle $\alpha_{i}$ between the orientation vector of the $i$ th NVC and the external magnetic field $\vec{H}$. Therefore the angle $\alpha_{i}$ serves as a convenient coordinate for characterizing the rotational diffusion of an NVC.

The NVC has $C_{3 v}$ symmetry, which implies the presence of four distinct orientation vectors in diamond crystal. Without loss of generality, if we choose our coordinate system such that one of the possible orientation vectors lies along the $+z$ axis, these four vectors will be given by

$$
\begin{aligned}
& \hat{d}_{1}=\hat{z}, \\
& \hat{d}_{2}=\frac{1}{3}(2 \sqrt{2} \hat{x}-\hat{z}), \\
& \hat{d}_{3}=-\frac{1}{3}(\sqrt{2} \hat{x}+\sqrt{6} \hat{y}+\hat{z}), \\
& \hat{d}_{4}=-\frac{1}{3}(\sqrt{2} \hat{x}-\sqrt{6} \hat{y}+\hat{z}),
\end{aligned}
$$

where $\hat{d}_{i}$ 's are all unit vectors. Note that the angle between any two distinct $\hat{d}_{i}$ 's is equal to $\sim 109.47^{\circ}$ [see Fig. 1 (a) for an illustration].

In order to calculate the fluorescence intensity of an NVC as a function of the angle it makes with the magnetic field, we need to calculate how the energy levels of the NVC depend on its orientation. To do so, we calculate the energy eigenvalues of the system by diagonalizing the Hamiltonian $\mathcal{H}$ given in Eq. (1). Defining the dimensionless variable $\xi=D /\left(3 H_{0} \gamma\right)$, we rewrite $\mathcal{H}$ as

$$
\mathcal{H}=H_{0}^{\prime}\left[\begin{array}{ccc}
\xi+\cos \alpha & \frac{1}{\sqrt{2}} \sin \alpha & 0 \\
\frac{1}{\sqrt{2}} \sin \alpha & -2 \xi & \frac{1}{\sqrt{2}} \sin \alpha \\
0 & \frac{1}{\sqrt{2}} \sin \alpha & \xi-\cos \alpha
\end{array}\right],
$$

(a)
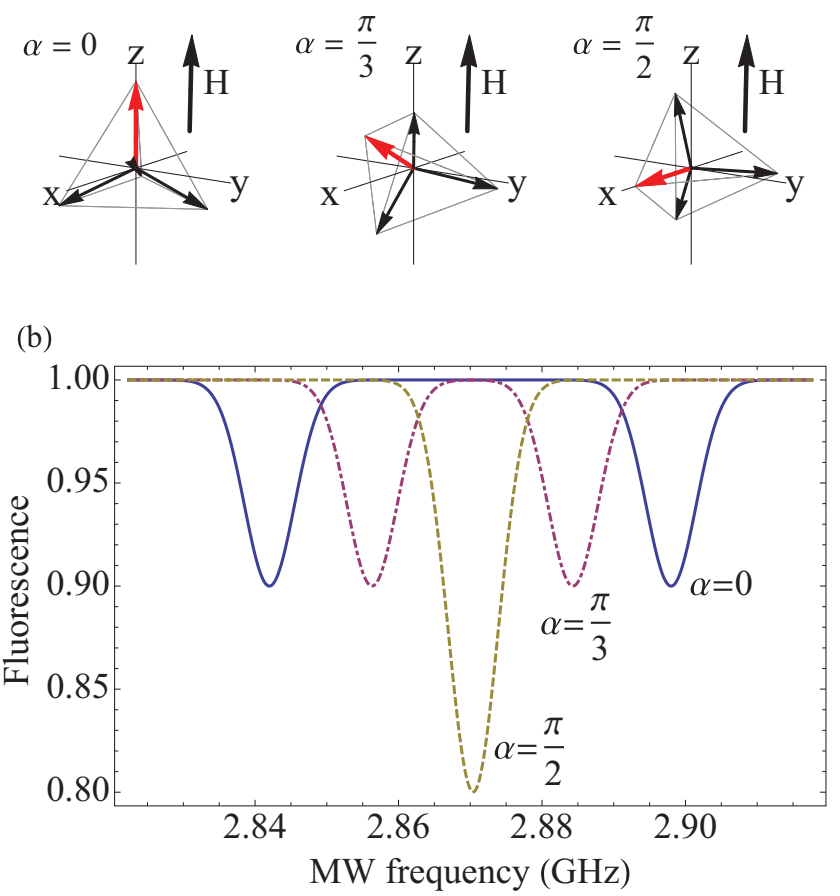

FIG. 1. (Color online) (a) Three examples illustrating different orientations of the NVC principle axis $\hat{d}$ (red arrow) and the magnetic field $\vec{H}$ (black arrow), where $\alpha$ is the angle between the two vectors. Other three NVC orientations that are crystallographically equivalent, $\langle 111\rangle$, are also depicted by black arrows in each tetrahedron. (b) The corresponding ODMR spectra for a single NVC. $D=2.87 \mathrm{GHz}$, $H_{z}=1 \mathrm{mT}, \varepsilon=0.1$ in Eq. (11) are assumed.

where $H_{0}^{\prime}=\gamma h H_{0}$, and we omit the subscript on $\alpha_{i}$, wherever the quantity pertains to a single NVC. Diagonalizing the matrix in Eq. (6), we obtain the following three eigenvalues:

$$
\begin{gathered}
\lambda_{1}=(2 / 3)^{\frac{1}{3}}\left(1+3 \xi^{2}+12^{-\frac{1}{3}} \zeta^{2}\right) \frac{H_{0}^{\prime}}{\zeta}, \\
\lambda_{2}=12^{-\frac{1}{3}} \frac{H_{0}^{\prime}}{\zeta}\left[\left(1+3 \xi^{2}\right)(-1-i \sqrt{3})+12^{-\frac{1}{3}}(-1+i \sqrt{3}) \zeta^{2}\right],
\end{gathered}
$$

$\lambda_{3}=12^{-\frac{1}{3}} \frac{H_{0}^{\prime}}{\zeta}\left[\left(1+3 \xi^{2}\right)(-1+i \sqrt{3})+12^{-\frac{1}{3}}(-1-i \sqrt{3}) \zeta^{2}\right]$,

where

$$
\begin{aligned}
\zeta= & \left\{9\left(-1+3 \cos ^{2} \alpha\right) \xi-18 \xi^{3}\right. \\
& +\sqrt{3}\left[-4+9\left(-1-18 \cos ^{2} \alpha+27 \cos ^{4} \alpha\right) \xi^{2}\right. \\
& \left.\left.-324 \xi^{4} \cos ^{2} \alpha\right]^{1 / 2}\right\}^{1 / 3} .
\end{aligned}
$$

If the NVC is oriented along the $+z$ axis, that is $\hat{d}=\hat{z}$, then the angle $\alpha$ is identical to the polar angle $\theta$ in spherical 
polar coordinates. For all other $\hat{d}$, we need to substitute $\cos \alpha=\vec{H} \cdot \hat{d} / H_{0}$.

The fluorescence signal from each NVC depends on the orientation of the diamond crystals with respect to the magnetic field. Based on our experimental observations, we consider the following empirical form for the fluorescence intensity of the $i$ th NVC:

$I_{i}\left(\alpha_{i}\right)=1-\epsilon\left[\exp \left(-\frac{\Delta E_{1, i}^{2}}{2 w^{2}}\right)+\exp \left(-\frac{\Delta E_{2, i}^{2}}{2 w^{2}}\right)\right]$,

where $\epsilon$ quantifies the amount of decrease in the fluorescence intensity due to magnetic resonance and $w$ quantifies the observed broadening of lines in the spectrum, and $\Delta E_{1, i}=$ $\lambda_{1, i}-\lambda_{2, i}-D, \Delta E_{2, i}=\lambda_{3, i}-\lambda_{2, i}-D$, in which $\lambda_{j, i}$ is the $j^{\text {th }}$ eigenvalue of the $i$ th NVC [see Eqs. (7)-(9)]. ${ }^{33}$ Since the NVC density is usually extremely low $\sim 0.1 \mathrm{ppm}$, we assume that there is no mutual interaction between NVCs. In this approximation, the total fluorescence intensity from $N$ NVCs, normalized to have a maximum value of 1 , is given by

$$
I(\vec{\alpha})=\frac{1}{N} \sum_{i=1}^{4} N_{i} I_{i}\left(\alpha_{i}\right),
$$

where $N_{i}$ is the number of NVCs with orientation vector $\hat{d}_{i}$, such that $\sum_{i} N_{i}=N$. Examples of ODMR spectra of single NVC for three different orientations $\alpha=0, \pi / 3$, and $\pi / 2$ are displayed in Fig. 1(b). As seen, the signal positions, which appear as dips in fluorescence intensity, are significantly different for different angles.

\section{B. Probability density function of a nanoparticle undergoing rotational diffusion}

We model the diamond nanoparticles as spherical rigid bodies in an isotropic three-dimensional (3D) viscous fluid. Diameters of the particles that are investigated in the laboratory are usually around several hundred nanometers. Through collisions, we suppose that the fluid molecules exert a random force on the particle. At equilibrium, this force is expected to be isotropic and $\delta$ correlated. Under these conditions, the translational and rotational motion of the particle can be characterized by Brownian motion, ${ }^{2}$ and the model we will present below applies. The probability density of finding such a particle with an orientation vector

$$
\hat{n}=\sin \theta \cos \phi \hat{x}+\sin \theta \sin \phi \hat{y}+\cos \theta \hat{z},
$$

is governed by the diffusion equation in spherical polar coordinates with $r=1^{34}$

$$
\begin{aligned}
& \frac{\partial \rho(\theta, \phi, t)}{\partial t} \\
& \quad=k_{\mathrm{d}}\left[\frac{1}{\sin \theta} \frac{\partial}{\partial \theta}\left(\sin \theta \frac{\partial \rho(\theta, \phi, t)}{\partial \theta}\right)+\frac{1}{\sin ^{2} \theta} \frac{\partial^{2} \rho(\theta, \phi, t)}{\partial \phi^{2}}\right],
\end{aligned}
$$

where $k_{\mathrm{d}}$ is the rotational diffusion coefficient (isotropic), such that $\rho(\theta, \phi, t) \sin \theta d \theta d \phi$ is the probability of finding the particle's orientation vector in the interval $[\theta+d \theta, \phi+d \phi]$ at time t. Through Stokes-Einstein relation, the diffusion coefficient can be expressed as $k_{\mathrm{d}}=k_{\mathrm{B}} T / 8 \pi r^{3} \eta$, where $k_{\mathrm{B}}$ is Boltzmann's constant, $r$ is the particle radius, and $\eta$ is the viscosity of the fluid. Equation (14) can be solved via separation of variables to yield

$$
\rho(\theta, \phi, t)=\sum_{\ell=0}^{\infty} \sum_{m=-\ell}^{\ell} C_{\ell m} Y_{\ell}^{m}(\theta, \phi) e^{-\ell(\ell+1) k_{\mathrm{d}} t},
$$

where $C_{\ell m}$ 's are determined by the initial conditions and $Y_{\ell}^{m}(\theta, \phi)$ 's are spherical harmonics

$$
Y_{\ell}^{m}(\theta, \phi)=\sqrt{\frac{2 \ell+1}{4 \pi} \frac{(\ell-m) !}{(\ell+m) !}} P_{\ell}^{m}(\cos \theta) e^{i m \phi},
$$

where $P_{\ell}^{m}(\theta)$ 's are associated Legendre polynomials. ${ }^{35}$ If we consider a localized initial condition such as

$$
\rho(\theta, \phi, t=0)=\frac{1}{\sin \theta_{0}} \delta\left(\theta-\theta_{0}\right) \delta\left(\phi-\phi_{0}\right),
$$

$C_{\ell m}$ 's will be equal to $Y_{\ell}^{m *}\left(\theta_{0}, \phi_{0}\right)$, where the superscript "*" denotes complex conjugation, and the solution for the probability density becomes

$$
\begin{aligned}
\rho\left(\theta, \phi, t \mid \theta_{0}, \phi_{0}\right) \\
\quad=\sum_{\ell=0}^{\infty} \sum_{m=-\ell}^{\ell} Y_{\ell}^{m *}\left(\theta_{0}, \phi_{0}\right) Y_{\ell}^{m}(\theta, \phi) e^{-\ell(\ell+1) k_{\mathrm{d}} t} .
\end{aligned}
$$

Note that the sum over $m$ could be written in an alternative form using the addition theorem for spherical harmonics: ${ }^{35}$

$$
\sum_{m=-\ell}^{\ell} Y_{\ell}^{m *}\left(\theta_{0}, \phi_{0}\right) Y_{\ell}^{m}(\theta, \phi)=\frac{2 \ell+1}{4 \pi} P_{\ell}\left(\hat{n}_{0} \cdot \hat{n}\right) .
$$

\section{Time autocorrelation function of the fluorescence intensity}

An experimentally accessible quantity which can be used to characterize the rotational diffusion of nanodiamonds is the time autocorrelation function of the fluorescence intensity, which we will denote as $\operatorname{ACF}(t)$. The $\operatorname{ACF}(t)$ for the fluorescence intensity $I(\theta, \phi)$ is given by

$$
\operatorname{ACF}(t)=\left\langle\left[I\left(\theta_{0}, \phi_{0}\right)-\mu_{0}\right][I(\theta, \phi)-\mu]\right\rangle,
$$

where $\left(\theta_{0}, \phi_{0}\right)$ and $(\theta, \phi)$ are the orientations of the particle at time 0 and $t$, respectively, and $\mu_{0}$ and $\mu$ are the average values of $I\left(\theta_{0}, \phi_{0}\right)$ and $I(\theta, \phi)$. Note that steady-state distribution of the particle's orientation is uniform over the ranges of $\theta$ and $\phi$, as rotation is isotropic, and the simple rotational diffusion we consider is a stationary process. We will calculate the autocorrelation function averaged over all initial conditions, which amounts to considering uniformly distributed initial conditions; hence, $\mu_{0}=\mu$. We note that the autocorrelation function can also be written as

$$
\operatorname{ACF}(t)=-\mu^{2}+\left\langle I\left(\theta_{0}, \phi_{0}\right) I(\theta, \phi)\right\rangle,
$$

as integrals of the following form vanish:

$$
\int_{0}^{\pi} d \theta \int_{0}^{2 \pi} d \phi \sin \theta Y_{\ell}^{m}(\theta, \phi),
$$

unless $\ell=0$. Evaluating Eq. (20) by using the definition given in Eq. (A2) (see Appendix A), we deduce that the 
autocorrelation function can be expressed in the following form:

$$
\operatorname{ACF}(t)=\sum_{\ell=2,4,6, \ldots}^{\infty} a_{\ell} e^{-\ell(\ell+1) k_{\mathrm{d}} t},
$$

where the coefficients $a_{\ell}$ are given by

$$
\begin{aligned}
a_{\ell}= & \frac{1}{4 \pi} \int d \Omega_{0} \int d \Omega \\
& \times \sum_{m=-\ell}^{\ell} Y_{\ell}^{m *}\left(\theta_{0}, \phi_{0}\right) Y_{\ell}^{m}(\theta, \phi) I\left(\theta_{0}, \phi_{0}\right) I(\theta, \phi), \\
= & \frac{2 \ell+1}{(4 \pi)^{2}} \int d \Omega_{0} \int d \Omega P_{\ell}\left(\hat{n}_{0} \cdot \hat{n}\right) I\left(\theta_{0}, \phi_{0}\right) I(\theta, \phi),
\end{aligned}
$$

and $\hat{n}$ is the unit vector in spherical polar coordinates given in Eq. (13). The term $\ell=0$ corresponds to the average of the squared intensity at the steady state [which is a uniform distribution, as seen from Eq. (18)], and is found to be equal to $\mu^{2}$. Nevertheless, values of the ACF at other times depend on all nonvanishing $a_{\ell}$, which are determined by the geometry of the problem (diffusion in a finite space) as well as all the physical parameters that control the fluorescence intensity. In Appendix A, we also provide the calculation of the time autocorrelation function for the orientation vector, which characterizes the rate at which information about the orientation of the particle is lost due to rotational diffusion.

\section{SIMULATION RESULTS}

In addition to the calculation of $\operatorname{ACF}(t)$ presented in the previous section, we also performed simulations of rotational diffusion of a nanodiamond modeled as a rigid body. We worked in the reference frame of the nanodiamond so that it is the magnetic field $\vec{H}$ that undergoes rotational diffusion. In our simulations, we rotate $\vec{H}$ around the three Cartesian coordinate axes by angles $\left(\Omega_{x}, \Omega_{y}, \Omega_{z}\right)$ generated randomly, all of which follow a normal distribution with the standard deviation $\sigma_{\mathrm{d}}^{2}=2 k_{d} \Delta t$, where $k_{\mathrm{d}}$ is the rotational diffusion constant and $\Delta t$ is the simulation time step.

The rotation of $\vec{H}$ at each simulation step was performed by using the rotation matrix derived by Beard and Schlick, ${ }^{36}$ which correctly takes into account the noncommuting nature of rotation operators in Langevin and Brownian dynamics simulations (see Appendix B). Successively applying the rotation matrix on $\vec{H}$, we generated time trajectories of the fluorescence intensity via Eq. (12). Once the fluorescence intensity was obtained as a function of time, we calculated the $\operatorname{ACF}(t)$ via

$$
\operatorname{ACF}(n \Delta t)=\frac{1}{M-n} \sum_{i=1}^{M-n}\left(I_{i}-\mu\right)\left(I_{i+n}-\mu\right),
$$

where $M$ is the total number of time points, $\mu$ is the average intensity and $I_{i}$ is the intensity at time $i \Delta t$.

\section{A. A single NVC}

Thermal fluctuations cause a nanodiamond to undergo rotational diffusion such that the angle $\alpha$ between the external

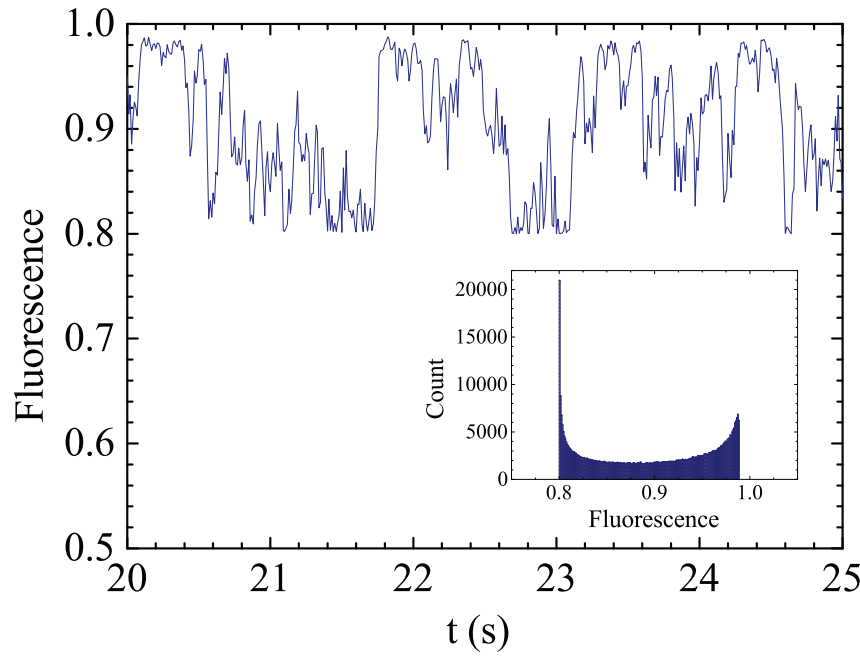

FIG. 2. (Color online) An example time trajectory of fluorescence intensity calculated for a single NVC at $H_{0}=0.3 \mathrm{mT}$. The reduction of fluorescence intensity through the intersystem crossing is assumed to be $20 \%$ [corresponding to $\epsilon=0.1$ in Eq. (11)], the sampling period was $\Delta t=0.01 \mathrm{~s}$, and the rotational diffusion coefficient $k_{\mathrm{d}}$ was set to $1 \mathrm{rad}^{2} \mathrm{~s}^{-1}$, such that $\sigma_{\mathrm{d}}^{2}=2 k_{\mathrm{d}} \Delta t=0.02 \mathrm{rad}^{2}$. $w=3.5 \mathrm{MHz}$. In the presence of more than one NVC, with different orientations, the fluorescence signal becomes smoother due to the increased frequency of detection at $f_{\mathrm{MW}}=2.87 \mathrm{GHz}$. (Inset) Histogram of the whole time trajectory.

magnetic field $\vec{H}$ and the NVC axis changes in time, as illustrated in Fig. 1. Simulation data showing the time-dependent spectrum that results from this rotational motion is displayed in Fig. 2. In Fig. 2, we plot the fluorescence intensity $I(t)$ for a single NVC as a function of time, measured at the microwave frequency $f_{\mathrm{MW}}=2.87 \mathrm{GHz}$. We envisage experiments where continuous laser illumination at $532 \mathrm{~nm}$ and microwave irradiation at $2.87 \mathrm{GHz}$ are employed, and the fluorescence intensity is periodically measured at a fixed frequency. The largest reduction in fluorescence intensity was observed at $\alpha=\pi / 2$ and around $f_{\mathrm{MW}}=2.87 \mathrm{GHz}$, which is equal to the zero-field gap $D$ [see the Hamiltonian (1)]. The intensity quickly returns to its background value once $\alpha$ deviates from $\pi / 2$. The inset of Fig. 2 displays a histogram of $I(t)$, showing the relative frequency with which the fluorescence signal was observed at a certain value. Note that the maximum intensity $\simeq 0.99$ is smaller than the base intensity which was set to unity, meaning that for these parameter values there is a finite contribution from the overlapping tails of the two signal dips [see Fig. 1(b)] even when they are most separated at $\alpha=0$. The presence of this residual intensity is crucial for not losing the signal during the experimental observations. As Horowitz et al. ${ }^{15}$ concluded, the optimal combination of the monitored frequency $f_{\mathrm{MW}}$ and the magnitude of the external field $H_{0}$ to observe the motion of the particle without losing the signal is realized either at $f_{\mathrm{MW}}=2.87 \mathrm{GHz}$ under an external magnetic field of a few Gauss, ${ }^{37}$ or at $f_{\mathrm{MW}}$ of the downward slope of the signal. Throughout this work, we monitored the fluorescence intensity at $f_{\mathrm{MW}}=2.87 \mathrm{GHz}$.

Using our simulation method described at the beginning of this section, we considered a nanodiamond containing a 


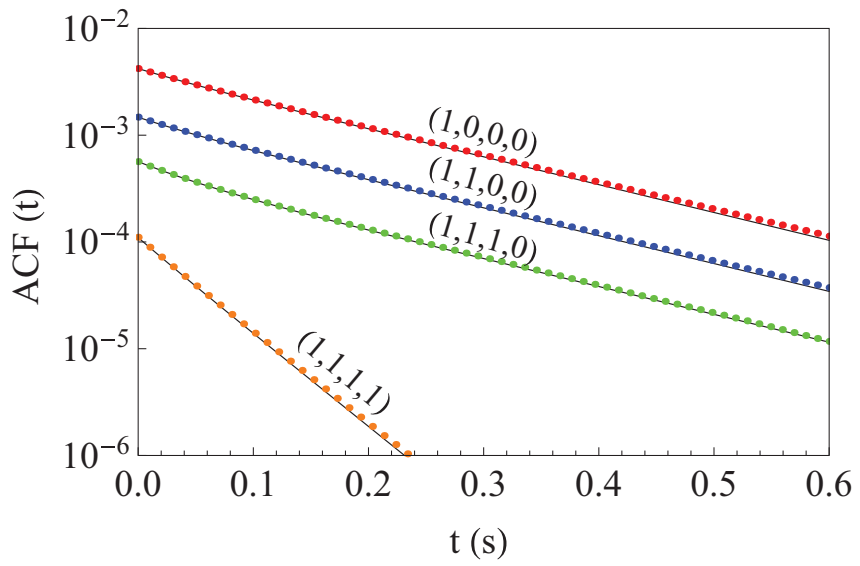

FIG. 3. (Color online) $\mathrm{ACF}(t)$ vs time, for different numbers of NVCs $N \leqslant 4$, where each NVC has a distinct orientation vector. The red, blue, and green data sets are for $N=1,2$, and 3, respectively, while the orange data set corresponds to $N=4$. NVC orientations were set according to the diamond crystal structure, given by the unit vectors in Eqs. (2)-(5). Forty independent trajectories $\operatorname{ACF}(t)$ of $10^{6}$ time points were calculated for each case. Parameter values are $k_{\mathrm{d}}=1 \mathrm{rad}^{2} \mathrm{~s}^{-1}$ and $H_{0}=0.3 \mathrm{mT}$.

single NVC and generated forty intensity ACF trajectories containing $10^{6}$ time points, with a time step of $\Delta t=0.01 \mathrm{~s}$, parameter values of $H_{0}=0.3 \mathrm{mT}, k_{\mathrm{d}}=1 \mathrm{rad}^{2} \mathrm{~s}^{-1}, \epsilon=0.1$, and $w=3.5 \mathrm{MHz}$. The result, obtained by averaging over all trajectories, is shown with the red curve in Fig. 3. In order to compare our theoretical results with the simulations, we also plot Eq. (23) together with the simulation data, keeping the first five nonvanishing exponential terms (see Appendix $\mathrm{C}$ for the values of the coefficients $a_{\ell}$ ).

\section{B. Multiple NVCs}

There are four possible, equivalent, orientations for an NVC in a diamond nanocrystal, as we noted earlier (see Fig. 1). We first examined the effect of the presence of multiple NVCs on the behavior of the ACF by considering three cases with $N=2,3$, and 4 NVCs, where each NVC points in a different direction. Similar to what we did for a single NVC, we generated forty ACF trajectories of $10^{6}$ time points, and averaged over all trajectories to obtain the $\mathrm{ACF}(t)$ curves displayed in Fig. 3. Again, we see excellent agreement with the prediction of Eq. (23) and the simulation data. Note that the results for nanodiamonds containing one, two and three NVCs are not significantly different from each other, except that the overall amplitude of the ACF decreases with the number of NVCs. For these three cases, we find that the asymptotic decay rate of the ACF is given by $6 k_{\mathrm{d}}$. However, we find that a nanodiamond containing four NVCs has a markedly different ACF, as its asymptotic decay rate is different from the rest, being $20 k_{\mathrm{d}}$. We attribute this to the symmetry of the configuration when all possible NVC states are occupied, and all NVCs have distinct orientations, which leads to a vanishing coefficient $a_{2}$ [see Eq. (24) and its numerical evaluation in Table I].

Next, we investigated the effect of degenerate NVC configurations, where there are multiple NVCs pointing in the

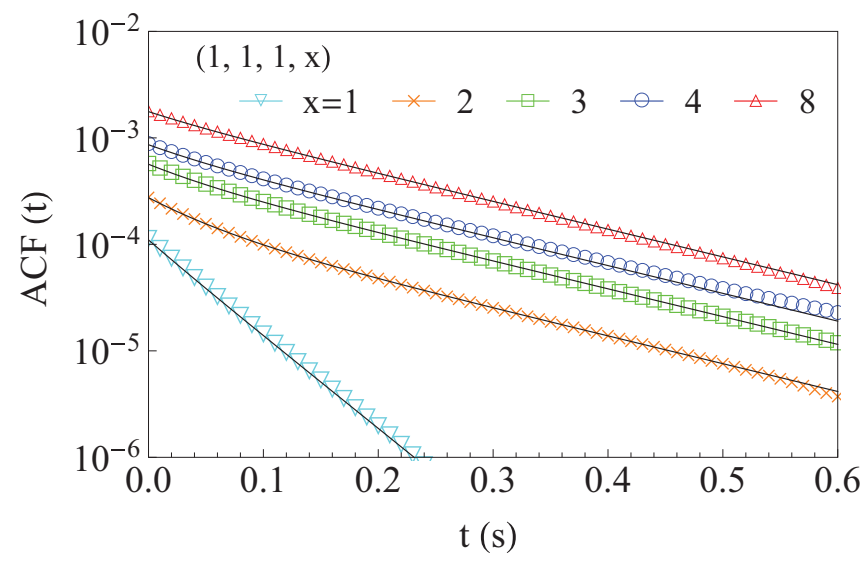

FIG. 4. (Color online) $\mathrm{ACF}(t)$ vs time, for different NVC configurations with four or more NVCs. The dots represent simulation results, and the curves are analytical results based on Eq. (23), showing excellent agreement between the simulation and theory. Different data sets correspond to NVC configurations of $(1,1,1, x)$, where $x$ is indicated in the legend. Parameter values are $k_{\mathrm{d}}=1 \mathrm{rad}^{2} \mathrm{~s}^{-1}$ and $H_{0}=0.3 \mathrm{mT}$.

same direction. In practice, this consideration is relevant for nanodiamonds with diameters larger than $100 \mathrm{~nm}$, where a few tens of NVCs are likely present. We considered NVC configurations of the form $\left(N_{1}, N_{2}, N_{3}, N_{4}\right)$ where $N_{i}$ denotes the number of NVCs with orientation vector $\hat{d}_{i}$ present in the nanodiamond. Here, we report our results for several specific configurations of the form $(1,1,1, x)$, where $x \in$ $\{2,3,4,8\}$. Simulation results as well as theoretical predictions [see Eq. (23)] are plotted in Fig. 4, also including the case $(1,1,1,1)$ that we discussed above, for comparison. First, we note that as soon as the symmetry of the configuration with $x=1$ is broken by adding one more NVC with an orientation $\hat{d}_{4}$, the asymptotic decay rate of $6 k_{\mathrm{d}}$ is recovered, which is the lowest possible value, as the decay rate is restricted to the values $\ell(\ell+1) k_{\mathrm{d}}$, where $\ell=2,4,6, \ldots$ Second, unlike the previous case with no degeneracy, increasing the number of NVCs result in an overall increase in the amplitude of the ACF.

In addition, we would like to note in passing that the decay rates for several artificial cases where NVCs are co-planar, or pointing along Cartesian axes in three-dimensions were also found to be significantly different. For instance, as shown in Fig. 5, the decay rate for two and six NVCs that are separated by $90^{\circ}$ and $60^{\circ}$ in the same plane were found to be $\sim 6.3 k_{\mathrm{d}}$ and $\sim 6.0 k_{\mathrm{d}}$, respectively, by fitting the tail part of the simulation data with a single exponential function. Whereas for the case of three and six orthogonal NVCs pointing along Cartesian axes, we observed a decay rate of $\sim 20.2 k_{\mathrm{d}}$. This suggests the importance of dimensionality of the structure which NVCs form in space. Nevertheless, we would like to note that even though the results in Fig. 5 suggest that the asymptotic decay constant for coplanar and noncoplanar arrangement of NVCs significantly differ, our previous result for three NVCs (see Fig. 3) does not support such a general conclusion, since a nanodiamond with three noncoplanar NVCs still has an asymptotic decay constant equal to those of the coplanar, artificial configurations in Fig. 5. 


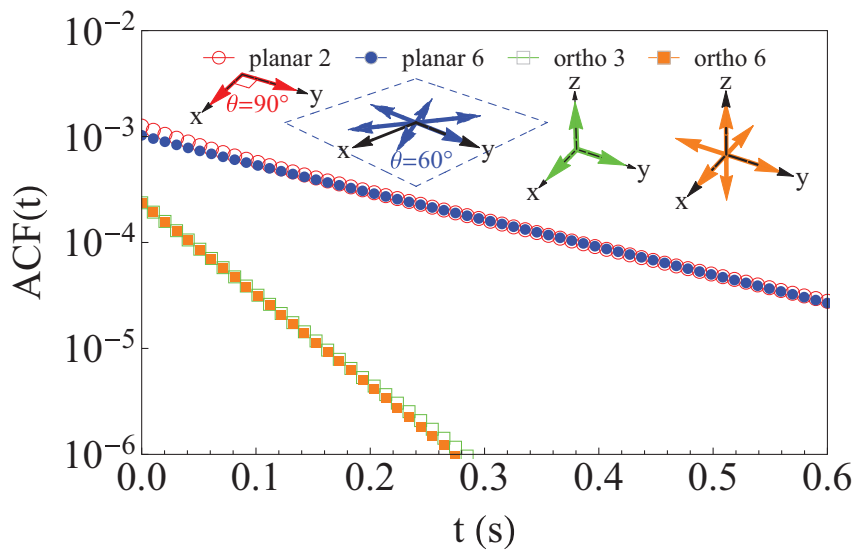

FIG. 5. (Color online) $\mathrm{ACF}(t)$ vs time, for several artificial configurations. Red and blue points correspond to co-planar NVCs, whereas green and orange points correspond to NVCs that point along Cartesian axes in three dimensions. See text for further explanation. To obtain each curve, forty independent trajectories $\operatorname{ACF}(t)$ of $10^{6}$ time points were calculated for each case. Parameter values are $k_{\mathrm{d}}=1 \mathrm{rad}^{2} \mathrm{~s}^{-1}$ and $H_{0}=0.3 \mathrm{mT}$.

\section{CONCLUDING REMARKS}

In this paper, we presented our theoretical and computational results on the relationship between the number and orientation of NVCs and the rotational diffusion coefficient of a nanodiamond. Supposing that the rotational motion of the nanodiamond can be approximated by simple Brownian diffusion, we derived an exact relationship between the fluorescence ACF of a particle, measured via ODMR, and its rotational diffusion coefficient. We clarified how the fluorescence ACF depends on the configuration of NVCs inside a nanodiamond, and verified our findings by performing simulations. Our results show that the fluorescence ACF can be expressed as a sum of exponential terms [see Eq. (23)], where the exponents are given by $-\ell(\ell+1) k_{\mathrm{d}} t$ with $\ell=2,4,6, \ldots$ While the exponents are purely determined by the nature of Brownian motion in isotropic 3D space, the coefficients contain information about the configuration of NVCs in the nanodiamond.

Due to the simplicity in interpreting observations, most of the experiments with nanodiamonds have employed particles that exclusively contain a single NVC. We believe that our results can be useful in extracting information from particles that contain more than one NVC, since we presented an analysis of how observables depend on more general NVC configurations (see Fig. 4). Among our findings, one case of particular interest is that if there are precisely four NVCs in a nanodiamond pointing in different directions, the coefficient for the $\ell=2$ term vanishes. Hence, the ACF decays much more rapidly compared to other (asymmetrical) NVC configurations, which can make it possible to identify nanodiamonds with this particular configuration. In addition to this qualitatively different result observed in the presence of four NVCs, we observed that the overall amplitude of the ACF depends on the number of NVCs, in a specific case where there is an excess number of NVCs in one of the orientations. When the NVC configuration is of the form $\left(n_{1}, n_{2}, n_{3}, n_{4}\right)$ where $n_{1}, n_{2}, n_{3}$ are 0 or 1 , and $n_{4} \geqslant 1$, we found two different trends in the absence and presence of degeneracy in NVC occupancy. As seen in Figs. 3 and 4, for $N \leqslant 4$, the overall amplitude of the ACF decreases with $N$, whereas the opposite is observed for $N \geqslant 4$, where there is an excess number of NVCs in one of the possible NVC orientations. Since the value of the ACF at $t=0$ is equal to the variance of the fluorescence intensity, the overall amplitude of the ACF is an indicator of the variability of the intensity signal. In the cases that we studied, it is found that the variability decreases until all NVC orientations are occupied nondegenerately, and starts increasing after that. Nevertheless, we would like to emphasize that it is the NVC orientation configuration rather than merely the number of NVCs that determines the behavior of the ACF, and more general NVC configurations with degeneracy can result in a different trend.

Depending on the time resolution of ODMR measurements, a single or multiexponential fit based on Eq. (23) can be performed on experimentally obtained ACFs to extract the decay coefficients. If the motion of nanodiamonds is wellcharacterized by isotropic Brownian rotation, one can then extract the rotational diffusion coefficient using the relationship between the exponential decay rates, and the $k_{\mathrm{d}}$. Note that when a relatively large nanodiamond containing hundreds of NVCs is employed, we can expect the NVC orientations to be uniformly distributed along four $\langle 111\rangle$ axes. Then, we could expect to use a single exponential fit to estimate $k_{d} \approx \tau_{c}^{-1} / 20$ to a good approximation. We also would like to point out that the choice of an appropriate magnitude for the magnetic field is essential for a reliable measurement of the fluorescence signal. An excessively strong magnetic field causes a large separation between the signal dips [see Fig. 1(b)], resulting in an increase in the fraction of time where the measured intensity corresponds to the maximum intensity (equal to unity), which does not contain any information about the rotation. Therefore, when the spectrum is monitored only within a narrow frequency window, this effect degrades the quality of the measured ACF. In general, the magnitude should be set around $0.3 \sim 0.5 \mathrm{mT},{ }^{37}$ also pointed in Ref. 15.

For applications, observing the rotational diffusion of nanodiamonds via ODMR can be a useful method to measure rheological and transport properties of a medium. For instance, in the simplest cases where linear response holds, mobility $\mu$ of a particle is related to its diffusion coefficient via $k_{\mathrm{d}}=\mu k_{\mathrm{B}} T$, where $k_{\mathrm{B}}$ is Boltzmann's constant. ${ }^{38}$ Therefore, deducing the diffusion coefficient from ODMR experiments performed at constant temperature can enable the extraction of particle mobility. When the geometry of the particle is known, one can then relate mobility to fluid viscosity via a generalized form of the Stokes-Einstein relationship. ${ }^{39}$ One interesting extension of this study would be generalizing the calculations to consider rotational motion in a viscoelastic medium, which can be applied to explore the properties of polymer melts and complex fluids such as the cytoplasm of a biological cell.

In our future studies, we would like to generalize the calculation of ACF to a case where the nanodiamond is practically confined to a two-dimensional space. A theoretical understanding of the rotation of particles confined to a surface is much needed to interpret observations on nanodiamonds that are incorporated to biological membranes. We believe 
that research along these lines will greatly contribute to our interpretation and design of new experiments to study the rotational motion of molecules that are confined to a membrane.

\section{ACKNOWLEDGMENTS}

This work was supported by Grant-in-Aid for Young Scientists (Start-up) 23810016 (Y.Y.), partly by grants (Y.H.) from NEXT Program of Japan Society for the Promotion of Science, and the World Premier International Research Center (WPI) Initiative of the Ministry of Education, Culture, Sports, Science and Technology (MEXT) of Japan.

\section{APPENDIX A: AUTOCORRELATION FUNCTION OF THE ORIENTATION VECTOR}

Here, we outline the calculation of the time it takes for the particle's orientation to relax to its equilibrium value, averaging over all initial conditions. The relaxation time can be obtained by calculating the time autocorrelation function of the orientation vector, given by

$$
\mathrm{ACF}_{0}(t)=\left\langle\hat{n}_{0} \cdot \hat{n}(t)\right\rangle
$$

where the angular brackets indicate averaging over the orientation vector at time $t$ as well as over all initial conditions uniformly such that

$$
\langle\cdot\rangle=\frac{1}{4 \pi} \int d \Omega_{0} \int d \Omega \rho\left(\theta, \phi, t \mid \theta_{0}, \phi_{0}\right)(\cdot),
$$

where $\Omega_{i}=d \theta_{i} d \phi_{i} \sin \theta_{i}$, and the integrations are performed over $0 \leqslant \theta_{i} \leqslant \pi$ and $0 \leqslant \phi_{i} \leqslant 2 \pi$. Note that the orientation vector averaged over all initial conditions or over the equilibrium distribution vanishes. Writing Eq. (A1) explicitly, we get

$$
\begin{aligned}
\mathrm{ACF}_{0}(t)= & \frac{1}{4 \pi} \int d \Omega_{0} \int d \Omega \sum_{\ell=0}^{\infty} \sum_{m=-\ell}^{\ell} Y_{\ell}^{m *}\left(\theta_{0}, \phi_{0}\right) \\
& \times Y_{\ell}^{m}(\theta, \phi) e^{-\ell(\ell+1) k_{\mathrm{d}} t}\left(\sin \theta \cos \phi \sin \theta_{0} \cos \phi_{0}\right. \\
& \left.+\sin \theta \sin \phi \sin \theta_{0} \sin \phi_{0}+\cos \theta \cos \theta_{0}\right),
\end{aligned}
$$

where we used Eqs. (13) and (18). Carrying out the integrals in Eq. (A3), we realize that only the terms with $\ell=1$ contribute to the final result, which is given by

$$
\operatorname{ACF}_{0}(t)=e^{-2 k_{\mathrm{d}} t} \text {. }
$$

Therefore the characteristic rotational relaxation time is $\tau_{0}=1 / 2 k_{\mathrm{d}}$.

\section{APPENDIX B: SIMULATION OF RANDOM ROTATIONS}

In order to rotate a rigid body around each axis by $\left(\Omega_{x}, \Omega_{y}, \Omega_{z}\right)$, the following rotation operator can be used: ${ }^{36}$

$$
\hat{R}\left(\Omega_{x}, \Omega_{y}, \Omega_{z}\right)=\left[\begin{array}{ccc}
\frac{\left(\Omega_{y}^{2}+\Omega_{z}^{2}\right) \cos \Omega+\Omega_{x}^{2}}{\Omega^{2}} & \frac{\Omega_{x} \Omega_{y}}{\Omega^{2}}(1-\cos \Omega)-\frac{\Omega_{z}}{\Omega} \sin \Omega & \frac{\Omega_{x} \Omega_{z}}{\Omega^{2}}(1-\cos \Omega)+\frac{\Omega_{y}}{\Omega} \sin \Omega \\
\frac{\Omega_{x} \Omega_{y}}{\Omega^{2}}(1-\cos \Omega)+\frac{\Omega_{z}}{\Omega} \sin \Omega & \frac{\left(\Omega_{x}^{2}+\Omega_{z}^{2}\right) \cos \Omega+\Omega_{y}^{2}}{\Omega^{2}} & \frac{\Omega_{y} \Omega_{z}}{\Omega^{2}}(1-\cos \Omega)-\frac{\Omega_{x}}{\Omega} \sin \Omega \\
\frac{\Omega_{x} \Omega_{z}}{\Omega^{2}}(1-\cos \Omega)-\frac{\Omega_{y}}{\Omega} \sin \Omega & \frac{\Omega_{y} \Omega_{z}}{\Omega^{2}}(1-\cos \Omega)+\frac{\Omega_{x}}{\Omega} \sin \Omega & \frac{\left(\Omega_{x}^{2}+\Omega_{y}^{2}\right) \cos \Omega+\Omega_{z}^{2}}{\Omega^{2}}
\end{array}\right],
$$

where $\Omega^{2}=\Omega_{x}^{2}+\Omega_{y}^{2}+\Omega_{z}^{2}$. This approach avoids the errors and bias introduced by applying rotation operators in sequence to rotate a body around different axis (rotation operators do not commute). See Beard and Schlick ${ }^{36}$ for details.

TABLE I. First few values of $a_{\ell}$ for different NVC configurations where the total number of NVCs is less than or equal to four and each NVC points in a different direction. We use the notation $\left(N_{1}, N_{2}, N_{3}, N_{4}\right)$ to denote that there are $N_{i}$ NVCs with the orientation vector $\hat{d}_{i}$. Parameter values are $H_{0}=3 \times 10^{-4} \mathrm{~T}, \gamma=28.02 \mathrm{GHz}$, $D=2.87 \mathrm{GHz}, \epsilon=0.1, w=3.5 \mathrm{MHz}$. Computed value of $a_{2}$ for four NVCs is comparable to the machine precision, which is $2.2204 \times 10^{-16}$, implying that $a_{2}$ vanishes in this case. For this data set, $\mu=\sqrt{a_{0}}=0.8973$.

\begin{tabular}{lcccc}
\hline \hline NVCs $\rightarrow$ & $(1,0,0,0)$ & $(1,1,0,0)$ & $(1,1,1,0)$ & $(1,1,1,1)$ \\
\hline$a_{2}$ & $3.79 \times 10^{-3}$ & $1.26 \times 10^{-3}$ & $4.21 \times 10^{-4}$ & $1.16 \times 10^{-19}$ \\
$a_{4}$ & $3.96 \times 10^{-4}$ & $2.00 \times 10^{-4}$ & $1.35 \times 10^{-4}$ & $1.02 \times 10^{-4}$ \\
$a_{6}$ & $2.03 \times 10^{-5}$ & $1.21 \times 10^{-5}$ & $9.37 \times 10^{-6}$ & $8.00 \times 10^{-6}$ \\
$a_{8}$ & $6.10 \times 10^{-7}$ & $2.22 \times 10^{-7}$ & $9.24 \times 10^{-8}$ & $2.76 \times 10^{-8}$ \\
$a_{10}$ & $1.20 \times 10^{-8}$ & $7.41 \times 10^{-9}$ & $5.87 \times 10^{-9}$ & $5.10 \times 10^{-9}$ \\
\hline \hline
\end{tabular}

\section{APPENDIX C: NUMERICAL VALUES OF COEFFICIENTS AND EXPONENTS THAT APPEAR IN THE ACF}

For a particular set of experimentally relevant parameters, values of the first few $a_{\ell}$ 's are given in Tables I and II as a function of the number of NVCs. Table I includes $a_{\ell}$ 's for cases where each NVC points in a different direction and there are up to four NVCs. Note that when there are four NVCs pointing in different directions, the coefficient $a_{2}$ vanishes. For some cases where there are more than four NVCs, such that there

TABLE II. First few values of $a_{\ell}$ in the presence of more than four NVCs, where at least four NVCs have distinct orientation vectors. Parameter values and the convention in labeling NVC numbers and orientations are the same as in Table I. Note that the coefficient $a_{2}$ does not vanish when there are 2 NVCs pointing in $\hat{d}_{4}$.

\begin{tabular}{lcccc}
\hline \hline NVCs $\rightarrow$ & $(1,1,1,2)$ & $(1,1,1,3)$ & $(1,1,1,4)$ & $(1,1,1,8)$ \\
\hline$a_{2}$ & $1.52 \times 10^{-4}$ & $4.21 \times 10^{-4}$ & $6.96 \times 10^{-4}$ & $1.53 \times 10^{-3}$ \\
$a_{4}$ & $1.14 \times 10^{-4}$ & $1.35 \times 10^{-4}$ & $1.56 \times 10^{-4}$ & $2.21 \times 10^{-4}$ \\
$a_{6}$ & $8.50 \times 10^{-6}$ & $9.37 \times 10^{-6}$ & $1.02 \times 10^{-5}$ & $1.30 \times 10^{-5}$ \\
$a_{8}$ & $5.09 \times 10^{-8}$ & $9.24 \times 10^{-8}$ & $1.35 \times 10^{-7}$ & $2.63 \times 10^{-7}$ \\
$a_{10}$ & $5.39 \times 10^{-9}$ & $5.89 \times 10^{-9}$ & $6.40 \times 10^{-9}$ & $7.95 \times 10^{-9}$ \\
\hline \hline
\end{tabular}


are more than one NVCs pointing in certain directions, we display the first few $a_{\ell}$ 's in Table II. Note that in the presence of degeneracy, none of the even coefficients up to $\ell=10$ vanish. *yyoshinari@icems.kyoto-u.ac.jp

†zkalay@icems.kyoto-u.ac.jp

${ }^{\ddagger}$ harada.yoshie.4r@kyoto-u.ac.jp

${ }^{1}$ P. W. van der Pas, Scientiarum Historia 13, 27 (1971).

${ }^{2}$ A. Einstein, Ann. Phys. 17, 549 (1905).

${ }^{3}$ M. von Smoluchowski, Ann. Phys. 21, 756 (1906).

${ }^{4}$ T. Li, S. Kheifets, D. Medellin, and M. G. Raizen, Science 328, 1673 (2010).

${ }^{5}$ T. Franosch, M. Grimm, M. Belushkin, F. M. Mor, G. Foffi, L. Forro, and S. Jeney, Nature (London) 478, 85 (2011).

${ }^{6}$ A. Jannasch, M. Mahamdeh, and E. Schäffer, Phys. Rev. Lett. 107, 228301 (2011).

${ }^{7}$ B. Wang, J. Kuo, S. C. Bae, and S. Granick, Nat. Mater. 11, 481 (2012).

${ }^{8}$ A. Ohlinger, A. Deak, A. A. Lutich, and J. Feldmann, Phys. Rev. Lett. 108, 018101 (2012).

${ }^{9}$ K. V. Edmond, M. T. Elsesser, G. L. Hunter, D. J. Pine, and E. R. Weeks, Proc. Natl. Acad. Sci. U.S.A. 109, 17891 (2012).

${ }^{10}$ J. S. Hyde and D. D. Thomas, Ann. Rev. Phys. Chem. 31, 293 (1980).

${ }^{11}$ D. D. Thomas, in Techniques for the Analysis of Membrane Proteins, edited by C. I. Ragan and R. J. Cherry (Springer, Netherlands, 1986), pp. 377-431.

${ }^{12}$ Y. Chang, H. Lee, K. Chen, C. Chang, D. Tsai, C. Fu, T. Lim, Y. Tzeng, C. Fang, C. Han, H. Chang, and W. Fann, Nat. Nanotechnol. 3, 284 (2008).

${ }^{13}$ I. Aharonovich, A. D. Greentree, and S. Prawer, Nat. Photon. 5, 397 (2011).

${ }^{14}$ L. P. McGuinness, Y. Yan, A. Stacey, D. A. Simpson, L. T. Hall, D. Maclaurin, S. Prawer, P. Mulvaney, J. Wrachtrup, F. Caruso, R. E. Scholten, and L. C. L. Hollenberg, Nat. Nanotechnol. 6, 358 (2011).

${ }^{15}$ V. R. Horowitz, B. J. Alemán, D. J. Christle, A. N. Cleland, and D. D. Awschalom, Proc. Natl. Acad. Sci. U.S.A. 109, 13493 (2012).

${ }^{16}$ M. Geiselmann, M. L. Juan, J. Renger, J. M. Say, L. J. Brown, F. J. G. de Abajo, F. Koppens, and R. Quidant, Nat. Nanotechnol. 8, 175 (2013).

${ }^{17}$ A. Gruber, A. Dräbenstedt, C. Tietz, L. Fleury, J. Wrachtrup, and C. von Borczyskowski, Science 276, 2012 (1997).

${ }^{18}$ J. R. Maze, P. L. Stanwix, J. S. Hodges, S. Hong, J. M. Taylor, P. Cappellaro, L. Jiang, M. V. Gurudev Dutt, E. Togan, A. S. Zibrov, A. Yacoby, R. L. Walsworth, and M. D. Lukin, Nature (London) 455, 644 (2008).

${ }^{19}$ G. Balasubramanian, I. Y. Chan, R. Kolesov, M. Al-Hmoud, J. Tisler, C. Shin, C. Kim, A. Wojcik, P. R. Hemmer, A. Krueger, T. Hanke, A. Leitenstorfer, R. Bratschitsch, F. Jelezko, and J. Wrachtrup, Nature (London) 455, 648 (2008).

${ }^{20}$ E. van Oort and M. Glasbeek, Chem. Phys. Lett. 168, 529 (1990).

${ }^{21}$ F. Dolde, H. Fedder, M. W. Doherty, T. Nöbauer, F. Rempp, G. Balasubramanian, T. Wolf, F. Reinhard, L. C. L. Hollenberg, F. Jelezko, and J. Wrachtrup, Nat. Phys. 7, 459 (2011).
${ }^{22}$ L. C. Bassett, F. J. Heremans, C. G. Yale, B. B. Buckley, and D. D. Awschalom, Phys. Rev. Lett. 107, 266403 (2011).

${ }^{23}$ V. M. Acosta, E. Bauch, M. P. Ledbetter, A. Waxman, L.-S. Bouchard, and D. Budker, Phys. Rev. Lett. 104, 070801 (2010).

${ }^{24}$ D. M. Toyli, C. F. de las Casas, D. J. Christle, V. V. Dobrovitski, and D. D. Awschalom, Proc. Natl. Acad. Sci. U.S.A. 110, 8417 (2013).

${ }^{25}$ P. Neumann, I. Jakobi, F. Dolde, C. Burk, R. Reuter, G. Waldherr, J. Honert, T. Wolf, A. Brunner, J. H. Shim, D. Suter, H. Sumiya, J. Isoya, and J. Wrachtrup, Nano Lett. 13, 2738 (2013).

${ }^{26}$ S. Steinert, F. Dolde, P. Neumann, A. Aird, B. Naydenov, G. Balasubramanian, F. Jelezko, and J. Wrachtrup, Rev. Sci. Instrum. 81, 043705 (2010).

${ }^{27}$ D. Le Sage, K. Arai, D. R. Glenn, S. J. DeVience, L. M. Pham, L. Rahn-Lee, M. D. Lukin, A. Yacoby, A. Komeili, and R. L. Walsworth, Nature (London) 496, 486 (2013).

${ }^{28}$ B. J. Maertz, A. P. Wijnheijmer, G. D. Fuchs, M. E. Nowakowski, and D. D. Awschalom, Appl. Phys. Lett. 96, 092504 (2010).

${ }^{29}$ M. P. Ledbetter, K. Jensen, R. Fischer, A. Jarmola, and D. Budker, Phys. Rev. A 86, 052116 (2012).

${ }^{30}$ D. Maclaurin, L. T. Hall, A. M. Martin, and L. C. L. Hollenberg, New J. Phys. 15, 013041 (2013).

${ }^{31}$ M. Böhmer and J. Enderlein, J. Opt. Soc. Am. B 20, 554 (2003).

${ }^{32}$ O. Faklaris, D. Garrot, V. Joshi, J. P. Boudou, T. Sauvage, P. Curmi, and F. Treussart, J. Eur. Opt. Soc. Rap. Public. 4, 09035 (2009).

${ }^{33}$ In experiments (including those conducted in our laboratory), spectral lines are broadened by a number of known and unknown factors, and the shape of the broadened lines can often be well described by a Gaussian or Lorentzian function. For the spectral simulation, we usually convolve a $\delta$ function signal intensity by such a normalized broadening kernel to reproduce the observed spectrum. This is equivalent to using a type of Eq. (11). In this work, we based our calculation on the more common Gaussian shape, resulting in Eq. (11).

${ }^{34} \mathrm{H}$. Risken, The Fokker-Planck Equation: Methods of Solution and Applications (Springer, Berlin, 1996).

${ }^{35} \mathrm{G}$. B. Arfken, H. J. Weber, and F. E. Harris, Mathematical Methods for Physicists: A Comprehensive Guide, 6th ed. (Academic Press, New York, 2005).

${ }^{36}$ D. Beard and T. Schlick, Biophys. J. 85, 2973 (2003).

${ }^{37}$ In experiments, the width of the fluorescence dips (see Fig. 1) tends to be different for each particle. The optimized magnetic field amplitude allowing us to measure the time trajectory of fluorescence (while keeping the time spent by measuring solely background signal at a minimum) is proportional to the separation of the dips due to the Zeeman effect, nearly the same as the full width at half maximum of the signal dip.

${ }^{38}$ L. D. Landau and E. M. Lifshitz, Statistical Physics, Part 1, 3rd ed. (Butterworth-Heinemann, Boston, 1980).

${ }^{39}$ T. M. Squires and T. G. Mason, Annu. Rev. Fluid Mech. 42, 413 (2010). 